\title{
Fast and Accurate Ultrasonography for Visceral Fat Measurement
}

\author{
You Zhou $^{1}$, Norihiro Koizumi ${ }^{1,2}$, Naoto Kubota ${ }^{2,3}$, Takaharu Asano ${ }^{3}$, \\ Kazuhito Yuhashi ${ }^{3}$, Takashi Mochizuki ${ }^{4}$, Takashi Kadowaki ${ }^{2,3}$, \\ Ichiro Sakuma $^{1,2}$, and Hongen Liao ${ }^{1,2}$ \\ ${ }^{1}$ Graduate School of Engineering, The University of Tokyo \\ ${ }^{2}$ Translational Systems Biology and Medicine Initiative, The University of Tokyo \\ ${ }^{3}$ Graduate School of Medicine, The University of Tokyo \\ ${ }^{4}$ Aloka Co. Ltd., Japan \\ \{zhouyou, sakuma, liao\}@ibmpe.t.u-tokyo.ac.jp
}

\begin{abstract}
Visceral fat area (VFA) has close relationship with hypertension, diabetes and cardiovascular disease, and therefore serve as a reliable indicator of these diseases. Abdominal computed tomography (CT) enables precise quantification of the VFA and has been considered as the gold standard for VFA assessment. In this paper, we develope a novel method to quickly and accurately measure the VFA with ultrasonography (US). We evaluated the novel method on five volunteers and the diagnosis procedures lasted less than 30 seconds averagely. The simulation results by our method were compared with VFA estimated by abdominal CT. The correlation coefficient between them was 0.913 for men and 0.858 for women. And the mean deviation of between VFA by $\mathrm{CT}$ and by our method was $19.8 \mathrm{~cm}^{2}$ for men and $13.3 \mathrm{~cm}^{2}$ for women.
\end{abstract}

\section{Introduction}

The term metabolic syndrome (MS), a combination of medical disorders that increase the risk of chronic diseases such as diabetes, hypertension, and cardiovascular disease, has been adopted by International Diabetes Federation (IDF) in 2006. MS is a major public health problem, the prevalence of which has increased worldwide. Visceral obesity, the excess accumulation of visceral fat deposits mainly around the waist, is thought to be a fundamental pathology for MS in particular. Therefore, accurate measurement of visceral fat represents an important tool in assessing MS.

Visceral fat, also known as organ fat, packes in between internal organs and the torso, as opposed to subcutaneous fat which is found underneath the skin. Since visceral fat is located deep inside the body and mixed with other organs, its accurate assessment has prooven to be challenging work. Abdominal computed tomography (CT) has been considered the most accurate and reproducible technique of body fat measurement, particularly abdominal adipose tissue. Abdominal CT enables accurate quantification of the visceral fat area (VFA) and therefore serves as the gold standard for visceral fat assessment [1]. However, abdominal CT has many drawbacks, including exposure to radiation, lack of simplicity, high cost, and time-consuming. 
Due to these limitations, a variety of alternative methods are being used to assess visceral fat amount and distribution. Previous studies have shown that waist circumference (WC), and WC-based indices can perform as some indicators of the level of visceral obesity [2]. These measurements are recommended as a simpler and easier screening method. However, due to the lack of the individual visualization of visceral fat, these methods have fatal drawbacks such as inability to distinguish subcutaneous fat from visceral fat, inability to take into account an individual's specific information, low level of reproducibility in the case of marked obesity, and most important, poor accuracy.

In recent years, simple methods for assessing visceral fat accumulation using ultrasonography (US) have been studied and were further confirmed by strong correlations with CT-detected VFA [3][4]. Ultrasound has many advantages such as non invasive to human bodies, low-cost, easy to be operated, real time and enables the visualization of visceral fat. Therefore, US-based methods show a balance on simplicity and accuracy between abdominal CT and WC-based indices. However, due to the poor image quality and limited visual field of US, the results of the existing US-based methods are still unsatisfactory and need further improvements.

In the study, a fast and accurate method is developed for visceral obesity studies to provided an estimation of the abdominal visceral fat area and distribution using ultrasonography.

\section{Method}

\subsection{Ultrasound Probe Compatible Device}

To provide a quick, easy-operated and accurate way to guide the ultrasonographic procedures, three factors are important: 1) Same positions and angles for ultrasound probe on different patients during diagnosis; 2) Distinct markers easy to be observable in US image; 3) Quantitive measurement on patients' curvature of abdomen.

We designed a belt-shaped ultrasound probe compatible device to fulfill these requirements (Fig.1). Two kinds of US measurements of visceral fat are taken. Firstly, US-determined visceral fat distance is defined as the distance between the internal face of the rectus abdominis muscle and the centre of the aorta from each diagnostic position. Secondly, the images acquire from the central position were saved for further processing.

Unlike CT, the ultrasound can only provide a limited scope inside one patient's body. The diagnosing angle and position of probe may differ from doctor to doctor due to their own experiences. This belt-shaped device provides doctors a standard which is easy to follow. In the visual field of an abdominal US, due to the flow of blood, the aorta is nearly the most distinct marker for US. Thus, the belt-shaped device is designed to be fixed in the exact position from where the aorta can be observed most clearly. As a result, the diagnosis process will be finished in seconds, which is convenient for patients as well. With an elastic material, this belt-shaped device would bend smoothly and mold to patient's abdomen. It is easy to understand that: a 
patient with mild visceral obesity in normal range of waist circumference would have a 'flatter' shape in abdomen, which means that the belt-shaped device will less bend when diagnosing, while a patient with more serious visceral obesity would get a 'plumper' waist, and resultingly more bent the belt-shaped device becomes when diagnosing. Hence, the curvature of the belt is proportional to the curvature of abdomen, which can serve as valuable characteristic of individual seriousness level of visceral obesity and give a reasonable classification of patients. With three probediagnosing positions rather than one, the curvature of abdomen can be described quantitively.

(a)



(b)

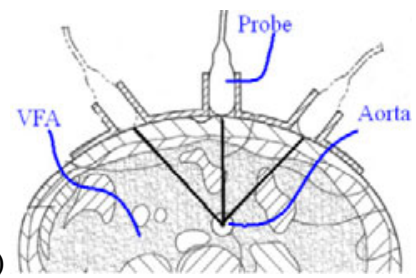

Fig. 1. Ultrasound probe compatible device. Distance and angle between two adjacent probes are $50 \mathrm{~mm}$ and $40^{\circ}$ in unbent situation. (a) Belt-shaped device design (b) Measurement method.

\subsection{Segmentation of Visceral Fat Area}

Analyzed results based on hundreds of abdominal CT images show that most visceral area of patient in his/her abdominal cross-section could be simulated as an ellipse, with aorta in the center. Although this approach may not be very exact for individual patient, the eccentricity of ellipse reflects the curvature of abdomen and therefore serves as a quantitive description of visceral obesity.

The ultrasound probe were detecting from three positions by the belt-shaped device. The information we can acquire contains: $b$, denotes the distance between the internal face of the rectus abdominis muscle and the centre of the aorta detected from front, and is defined as semi-minor axis of ellipse; $b^{\prime}$, denotes the distance between front-end of ultrasound probe in the central diagnosing position and the centre of the aorta detected from front, $r$, denotes the same distance detected from one side; $h^{\prime}$, denotes the distance between two probes $(50 \mathrm{~mm}) ; h$, denotes the curve length between $b$ and $r$ derived from $h^{\prime}$ by $h=h^{\prime} b / b^{\prime}$ as showed in Fig. 2(a). Then, by ellipse circumference and ellipse parametric equation, we can calculate: $a$, denotes the semi-major axis; $\theta$, denotes the angle between two probes.

For abdominal cross-section of a patient who has been diagnosed (denotes as patient*), we discretize the visceral area based on the ellipse approach as follow. The ellipse is divided uniformly by angle and axis length. Each section is identified by a number $i$. For patient*, we define the area of section $i$ as $a_{i}^{*}$, the fat percentage of section $i$ as $p_{i}^{*}$. Succinctly, we have $\boldsymbol{A}^{*}=\left(a_{1}^{*}, a_{2}^{*}, \ldots, a_{n}^{*}\right)^{T}$ and $\boldsymbol{P}^{*}=\left(p_{1}^{*}, p_{2}^{*}, \ldots, p_{n}^{*}\right)^{T}$, where $n$ refers to the total number of sections (In this paper, 
$n \triangleq 30 \times 40=1200$ ). Similar with a matrix, we can define $N_{i}^{*}$, the neighbourhood of $p_{i}^{*}$ as: the fat percentages of sections who share the same edges or vertices with section $i$ (Fig.2(b)). Immediately, there follows

$$
V F A=\boldsymbol{A}^{* T} \boldsymbol{P}^{*} \text {. }
$$

The computation of $\boldsymbol{A}^{*}$ is trivial and main difficulty is how to give a reasonable estimation of $\boldsymbol{P}^{*}$.

In order to distinguish the visceral fat from other organs, we used a Markov Random Field (MRF) based segmentation algorithm (Fig. 3). The main process of this algorithm follows [5]. We must declare here that this kind of algorithms were not developed specially for solving the fat recognition problem of ultrasonographic image, and have never been introduced into this field.

(a)

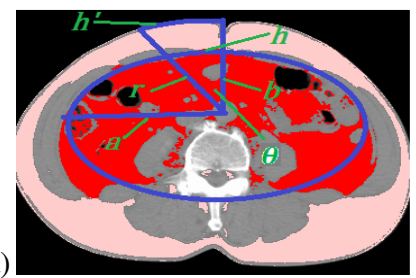

(b)

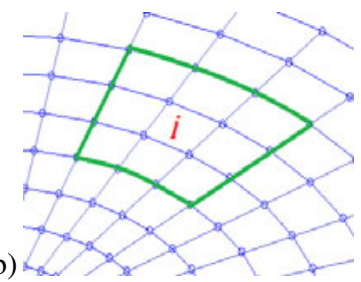

Fig. 2. Ellipse approach of abdominal cross-section. (a) Parameters of ellipse. Red part is the visceral fat. Grey part is other organs. (b) Neighbourhood in a discretized ellipse. Green part refers to $N_{i}^{*}$, the neighbourhood of $p_{i}^{*}$.

(a)

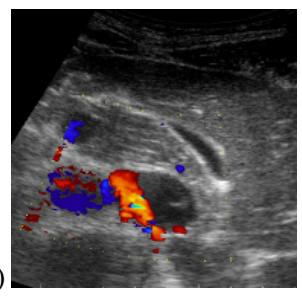

(b)



(c)

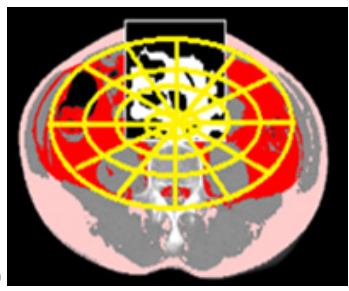

Fig. 3. Distinction of visceral fat. (a) Original US image. The colorful part in central indicates aorta. (b) Segmentation of visceral fat. The white part indicates the visceral fat area. (c) Matching US visual field into the whole visceral area.

\subsection{Matching with Pre- stored Data}

In this part, we compare the ultrasonographic image of patient*, with pre-stored CT scanned image of previous patients, and choose the patients whose situations are most similar with the current patient. We established a set $V$ from 146 patients abdominal CT scanned images in a urban hospital, which cover full gamut of patients in its community. This pre-stored CT scanned images served as a pre-stored database with which the current patient* can compare. 
The visual field of ultrasonography is only local. So, we match the ultrasonography into the whole visceral area, and divide it into $m$ sections following the previous part (Fig. 3(c)). We denote section $i$ has area $a_{i}^{*}$ and fat percentage $p_{i}^{*}$, for every $i=1,2, \ldots, m$. We have an estimation of $m$ components of $\boldsymbol{P}^{*}$, and the problem now is how to estimate the fat percentage of the remaining $(n-m)$ hidden sections $\boldsymbol{P}_{\boldsymbol{h}}^{*}=\left(p_{m+1}^{*}, p_{m+2}^{*}, \ldots, p_{n}^{*}\right)^{T}$. For CT images, the discretization of ellipse can be accomplished without difficulty. Then, section $i$ of patient $k$, has the fat percentage $P_{i}^{k}$. Naturally, there is $\boldsymbol{P}^{k}=\left(p_{1}^{k}, p_{2}^{k}, \ldots, p_{n}^{k}\right)^{T}$, for $k=1,2, \ldots, 146$.

Now, we want to find out the patients from $V$ whose situations are most similar with patient*. As a result, the visceral fat distributions of those patients would serve as references for patient $*$. We evaluate the correlative level between patient* and patient $k$ by matching $p_{1}^{*}, p_{2}^{*}, \ldots, p_{m}^{*}$ and $p_{1}^{k}, p_{2}^{k}, \ldots, p_{m}^{k}$. We use the Grey-Level Correlation Formula to accomplish it

$$
r^{k}=\frac{\sum_{i=1}^{m}\left(p_{i}^{*}-\overline{p^{*}}\right)\left(p_{i}^{k}-\overline{p^{k}}\right)}{\sqrt{\sum_{i=1}^{m}\left(p_{i}^{*}-\overline{p^{*}}\right)^{2} \sum_{i=1}^{m}\left(p_{i}^{k}-\overline{p^{k}}\right)^{2}}},
$$

where $\overline{p^{*}}$ and $\overline{p^{k}}$ refer to the average of $p_{1}^{*}, p_{2}^{*}, \ldots, p_{m}^{*}$ and $p_{1}^{k}, p_{2}^{k}, \ldots, p_{m}^{k}$ respectively. Before the matching process, the patients whose eccentricity $e$ and minor axis $b$ differ too large from patient* will be rejected in order to accelerate. The 10 patients with max value of $r^{k}$ compose the set $B$. The CT image of those chosen patients would serve as the basis of fat estimation of current patient*.

We expect that $\boldsymbol{P}_{\boldsymbol{h}}^{*}$ can be expressed as a 'combination' of patients from $B$ and this combination can reflect the visceral fat distribution of patient $*$. To achieve it, we establish an optimization standard. By (3), we defines the cost at one section, and (4) is the integrally cost, which should be minimized. Consider $N_{i}^{*} \subset \boldsymbol{P}^{*}$, the neighbourhood of $p_{i}^{*}$. We give a choice not only realizing optimization at single $p_{i}^{*}$ itself, but also throughout its neighbourhood $N_{i}^{*}$. We define the distance cost function between patient* and patient $k$ at section $i$ as

$$
\left(\left|p_{i}^{*}-p_{j}^{*}\right|-\left|p_{i}^{k}-p_{j}^{k}\right|\right)^{2}, p_{j}^{*} \in N_{i}^{*}, p_{j}^{k} \in N_{i}^{k},
$$

where $N_{i}^{*}$ and $N_{i}^{k}$ refers to the neighbourhood of $p_{j}^{*}$ and $p_{j}^{k}$ respectively. Hence, integrally, the $\boldsymbol{P}_{\boldsymbol{h}}^{*}$ should be the vector who satisfies that

$$
\begin{gathered}
\arg \min _{p_{i}^{*} \in \boldsymbol{P}_{\boldsymbol{h}}^{*}} \sum_{i=m+1}^{n} \sum_{k=1}^{10} \sum_{p_{j}^{*} \in N_{i}^{*}, p_{j}^{k} \in N_{i}^{k}}\left(\left|p_{i}^{*}-p_{j}^{*}\right|-\left|p_{i}^{k}-p_{j}^{k}\right|\right)^{2}, \\
p_{i}^{*} \in[0,1], \text { for } i=1,2, \ldots, n .
\end{gathered}
$$

Consequently, the determination of $\boldsymbol{P}_{\boldsymbol{h}}^{*}$ has been transformed into an optimization problem. This numerical optimization is performed automatically by a constraint nonlinear conjugate gradient method (CGM).

\section{Experiment Results}

In this part, all correlations between two variables were evaluated using Pearson's product-moment correlation coefficient. Comparisons between each two groups were done with an unpaired t-test. 


\subsection{Evaluation Experiment of US}

We tested the belt-shaped ultrasound probe compatible device on five volunteers from an urban community. All our ultrasonographic procedures were performed by the same examiner using a portable ultrasonography equipment ( $\alpha 10$, ALOKA, Japan). The center of the belt-shaped device fixed exactly $2 \mathrm{~cm}$ left to umbilicus for each patient. Each patient assumed a supine position, and US data were measured at the end of expiration by a 6-MHz 2D abdominal probe, while the probe was making contact with patients' skin as slight as possible (Fig.4).

For each volunteer, the diagnosis procedures lasted less than 30 seconds. This is quite an acceptable time during a medical examination. In addition, More quick diagnosis speed is also expectable in the future if doctors become more proficient in this novel method.
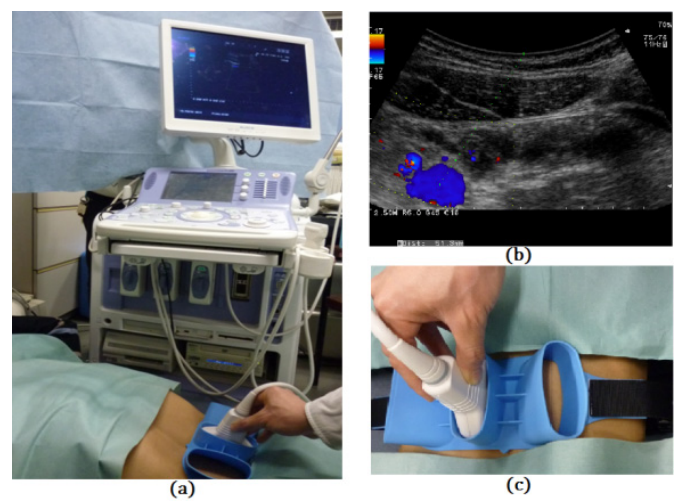

Fig. 4. Practical diagnosis procedures for a patient. (a) Diagnosis by US. (b) Distance of visceral fat measured in US image. (c) Belt-shaped ultrasound probe compatible device.

\subsection{Campare with CT Results}

Simulation on 146 patients' abdominal CT images were done and showed that there was strong correlation between the US observed VFA from front psotion and the total VFA of an individual patient. The coefficent was $r=0.883$ and the level of significance was p-value $<2.2 \mathrm{e}-16$ (Fig. 5). As a result, it is reasonable to use the US observed fat percentage as an indicator for total VFA.

Since abdominal CT holds the most accurate quantification of the VFA, we treated it as the testing standard for visceral fat assessment. Abdominal cross-section by CT scanning was obtained in a single tomographic slice at umbilical level as ultrasound probe did. Portions with a CT number of -200 to -10 Hounsfield Units (HU) were separated as adipose tissue and their areas were automatically calculated. 
We simulated our method on 28 male and 35 female samples, and compared the results with abdominal CT. Figure 6 shows the comparisons of CT and our US-based method. The mean deviation between VFA measured by CT and our US method for each patient is $19.8 \mathrm{~cm}^{2}$ for men and $13.3 \mathrm{~cm}^{2}$ for women. The correlation between these two groups is also presented. For men, the coefficent was $r=0.913$ and the level of significance was $\mathrm{p}$-value $=1.179 \mathrm{e}-11$. For women, there are $\mathrm{r}=0.858$ and $\mathrm{p}$-value $=4.674 \mathrm{e}-11$. These results proofed the significant positive correlations between VFA measured by CT and by our method.

And we also divided these 63 patients in both sex into three groups by the CTmeasured VFA: low ( $\leq 100 \mathrm{~cm}^{2}$ ), medium (between $100 \mathrm{~cm}^{2}$ and $140 \mathrm{~cm}^{2}$ ) and high $\left(>140 \mathrm{~cm}^{2}\right)$. The average and max VFA deviations between US method and CT method of these three groups were shown in Table 1. From this table, it is easy to notice that our US-based method performed better on the medium group than other two groups averagely. The reason of this is that the data per-stored data mainly laid mainly in this VFA range. Thus, the result reminds us that the pre-stored database is very essential for our method and it must cover full range of patients in enough quantities. On the other hand, the max VFA deviations are all too large in three groups. This demonstrates that there are many personal detailed characteristics of certain patients have been omitted in our method, which should be checked carefully for the further improvement of this US-based method.

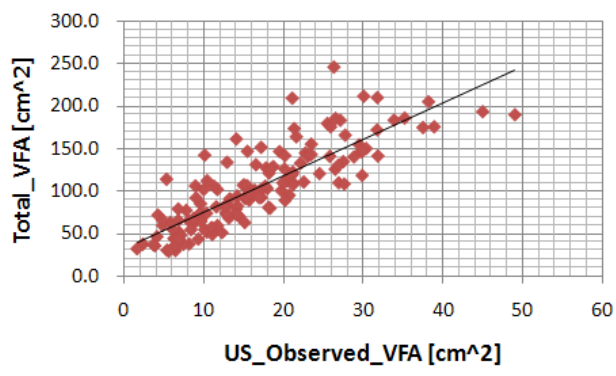

Fig. 5. Scattergram of relationship between US observed VFA and the total VFA
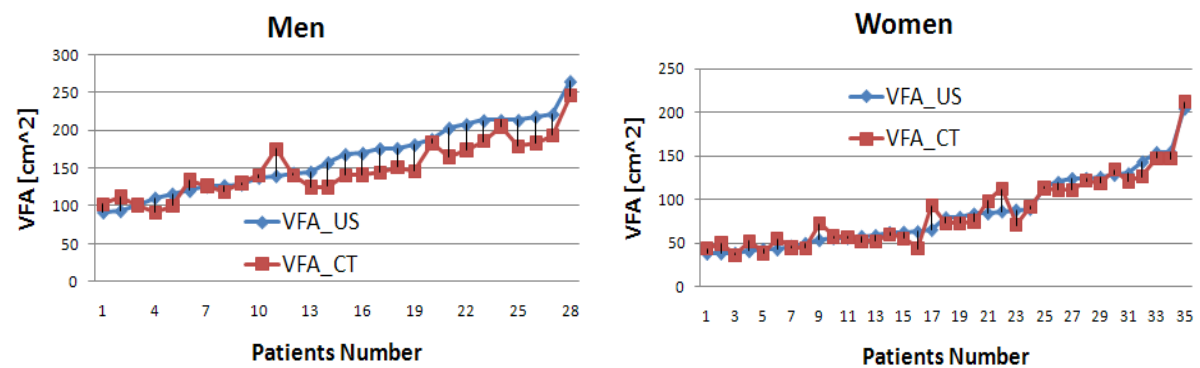

Fig. 6. Camprison of our US-based method and CT for men and women 
Table 1. VFA deviations between US_method and CT_method in different groups

\begin{tabular}{c|c|c|c|c}
\hline \multicolumn{2}{c|}{ Groups } & low & medium & high \\
\hline \multicolumn{2}{c|}{ Numbers of patients } & 17 & 25 & 21 \\
\hline \multirow{2}{*}{$\begin{array}{c}\text { VFA deviations } \\
\left(\mathrm{cm}^{2}\right)\end{array}$} & Average & 15.7 & 9.1 & 27.3 \\
\cline { 2 - 5 } & Max & 42.2 & 36.9 & 37.3 \\
\hline
\end{tabular}

\section{Discussions and Conclusion}

This paper proposes a novel US-based method for estimation of the VFA. A fast and convenient diagnosis method was introduced and a robust algorithm was proposed to estimate a patient's VFA from US data automatically. As far as we know, this is the first attempt to compute VFA by ultrasound, and our results were shown to be strongly correlated with CT measurements.

However, one main limitation of this present study is that all the data of patients were collected from the same ethnic group. Thus, the results cannot generalize directly to other individuals of certain ethnic groups. For instance, the female body type in particular differs largely between Westerners and Easterners. As a result, how to apply this novel method universally needs further discussions.

Till now, we have developed a US-based method whose accuracy reaches the level of abdominal CT. However, it is not an entirely impossible thing that the US-based method will become a more reliable indicator for MS than abdominal CT in the future. Due to the non-invasiveness and simplity of US, diagnosis at different levels of the abdomen will be accomplished without much difficulties. Consequencely, 3D individual visualization and estimation of visceral fat can be realized by US, while usually only one slice of abdominal cross-section image can be acquired by CT in medical examination due to the harmness of rediation. Secondly, due to its real-time characteristics, US allows us to detect visceral fat over a period of time. One patient's abdominal cross-sectional area when expiring will differ largely from inspiring. Abdominal CT cannot take this difference into consider and therefore would be less convincing than US in this respect. Further, by US, we may represent a useful method for monitoring weight loss, variations and transfer of visceral fat, which can be expected to indicate the associated risks of MS more accurately.

In conclusion, although its results are still rough and there are many details need discussing, the present US-based method has proofed to be a considerably fast and accurate way for VFA estimation.

\section{Acknowledgment}

This work was supported in part by Grant for Translational Systems Biology and Medicine Initiative (TSBMI) from the Ministry of Education, Culture, Sports, Science and Technology of Japan. 


\section{References}

1. Rossner, S., Bo, W.J., Hiltbrandt, E., et al.: Adipose tissue determinations in cadavers-a comparison between cross-sectional planimetry and computed tomography. Int. J. Obes. Relat. Metab. Disord. 14, 893-902 (1990)

2. Hsieh, S.D., Yoshinaga, H., Muto, T.: Waist-to-height ratio, a simple and practical index for assessing central fat distribution and metabolic risk in Japanese men and women. Int. J. Obes. Relat. Metab. Disord. 27, 610-616 (2003)

3. Chiba, Y., Saitoh, S., et al.: Relationship between Visceral Fat and Cardiovascular Disease Risk Factors: The Tanno and Sobetsu Study. Hypertension Research 30, 229-236 (2007)

4. Ribeiro-Filho, F.F., et al.: Methods of Estimation of Visceral Fat: Advantages of Ultrasonography. Obesity Research 11(12), 1488-1494 (2003)

5. Lorenz, A., Haas, C., Ermert, H.: Segmentation of ultrasonic prostate images using a probabilistic model based on Markov random processes. Ultrason Imaging 19, 44-45 (1997) 